\title{
MedienPädagogik
}

Zeitschrift für Theorie und Praxis der Medienbildung

\section{Gelingensbedingungen für den Einsatz digitaler Medien}

\section{Eine qualitative Studie in der Alphabetisierung und Grundbildung}

Ilka Koppel

\section{Zusammenfassung}

Ausgehend von einer tiefgreifenden Mediatisierung der Gesellschaft (vgl. z. B. Couldry und Hepp 2016) werden für die Teilhabe an der Gesellschaft neben der literalen und der mathematischen Grundbildung nun auch eine digitale Grundbildung notwendig (BMBF und KMK 2016; Tröster und Schrader 2016). Entsprechend wird eine stärkere Einbindung digitaler Technologien in der Erwachsenenbildung (European Comission 2015; Helbig und Hofhues 2018; Pietraß 2015) sowie eine Weiterbildungsoffensive gefordert und auch umgesetzt (vgl. z. B. Staatsministerium Baden-Württemberg). Auch wenn der Bedarf an digitaler Grundbildung hoch ist, wird der Erwachsenenbildung gegenüber computerbasierten Lernprozessen und deren konzeptionelle Einbindung eine distanzierte Einstellung (Arnold 2014, 157; Hartung-Griemberg 2017) postuliert. Insbesondere ist dies im Bereich der Alphabetisierung der Fall (Helbig und Hofhues 2018, 12). Aber gerade diese Zielgruppe kann besonders von dem Einsatz digitaler Medien profitieren (vgl. z. B. Koppel 2017; Wolf und Koppel 2017). Im Bereich der Alphabetisierung und Grundbildung existieren allerdings bisher kaum Erkenntnisse darüber, welche Faktoren zu einem gelingenden Einsatz digitaler Medien beitragen. Ziel des hier vorgestellten Projektes «Geco» ist es daher, mittels leitfaden- 
gestützter Interviews Gelingensbedingungen für den Einsatz computerbasierter Lernprogramme in der Alphabetisierung und Grundbildung zu identifizieren. Die Ergebnisse der Studie/des Projekts werden im Folgenden vorgestellt.

\title{
Key Success Factors Using Digital Media. Basic Research for Basic Adult Education
}

\begin{abstract}
Based on a far-reaching mediatisation of society (cf. e.g. Couldry and Hepp 2016), participation in society requires not only literary and mathematical basic education but also digital basic education (BMBF and KMK 2016; Tröster and Schrader 2016). Accordingly, a stronger integration of digital technologies in adult education (European Commission 2015; Helbig and Hofhues 2018; Pietraß 2015) and a further education offensive (Deutscher Volkshochschulverband e.V. 2015) by the German Adult Education Association are called for. Even if the demand for digital basic education is high, a distanced attitude is postulated (Arnold 2014 157; Hartung-Griemberg 2017) towards adult education in relation to computer-based learning processes and their conceptual integration. This is particularly the case in the area of literacy (Helbig and Hofhues 2018, 12). But it is this target group in particular that can benefit from the use of digital media (cf. e.g. Koppel 2017; Wolf and Koppel 2017). In the area of literacy and basic education, however, there is hardly any knowledge to date about which factors contribute to the successful use of digital media. The aim of the "Geco" project presented here is therefore to identify key success factors for the use of computerbased learning programs in basic education. The findings presented in this paper were obtained through guideline-based interviews and are presented below.
\end{abstract}




\section{Einführung}

Wir befinden uns in einem Prozess tiefgreifender Mediatisierung (vgl. z. B. Couldry und Hepp 2016). Digitale Medien werden sowohl für die Unterstützung von Lernprozessen ${ }^{1}$ als auch die Unterstützung von formalen Abläufen, beispielsweise bei Behörden, eingesetzt. Gleichzeitig wachsen damit die Anforderungen jeglicher Bevölkerungsgruppen an medienbezogene Kompetenzen (vgl. z. B. Petko 2006), sodass auch die Notwendigkeit, das Verständnis von Grundbildung um medienbezogene Kompetenzen zu erweitern (Niesyto und Moser 2018), steigt. Obwohl beispielsweise im Bereich der literalen Grundbildung 6,2 Millionen Personen ein Förderbedarf attestiert wird (Grotlüschen u. a. 2019, 5), besucht nur ein Bruchteil dessen (ca. 0,7 Prozent) Alphabetisierungskurse an Volkshochschulen ${ }^{2}$ (Reichart, Lux, und Huntemann 2018, 47). Den PISA-Ergebnissen nach ist davon auszugehen, dass trotz einer Verbesserung der Lesekompetenz seit der ersten PISA-Studie im Jahr 2000 jährlich immer noch ca. 14\% eines Altersjahrganges mit Lese-, Schreib-, und Rechenschwierigkeiten hinzukommen (Wolf und Koppel 2014). Dies ist nach aktuellem Forschungsstand in diversen Herausforderungen begründet (vgl. z. B. Bonna und Nienkämper 2011; Döbert, Hubertus, und Nickel 2000; Koppel 2017), für deren Begegnung digitale Medien aufgrund ihrer anonymen, ressourcenschonenden ${ }^{3}$, individuellen ${ }^{4}$ und selbstständigen Nutzbarkeit einen Beitrag zur Grundbildung leisten können.

Allerdings nutzen Personen mit geringer Literalität digitale Medien weniger als die Gesamtbevölkerung (Buddeberg und Grotlüschen 2020, 205 ff.) und verfügen daher auch über weniger Erfahrungen mit entsprechender Technik im Vergleich zu literalisierten Personen. So geben 14 Prozent

1 Der Mehrwert digitaler Medien wird hier nicht weiter ausgeführt. An dieser Stelle sei exemplarisch verwiesen auf Kerres (2009), Pietraß (2011) und (2017).

2 Die Volkshochschule ist der grösste Anbieter für Alphabetisierungs- und Grundbildungskurse. Schätzungsweise kommen zu den Teilnehmenden an VHS jährlich noch etwa 5000 Lernende in anderen Bildungsträgern hinzu (vgl. z. B. Ernst und Schneider 2009).

3 Gerade im Grundbildungsbereich ist mit analogen Medien oftmals eine 1:1-Betreuung notwendig.

4 Digitale Medien bieten diverse Potenziale für das individuelle Lernen, welches aufgrund der heterogenen Bildungsbiographien eine besondere Bedeutung im Grundbildungsbereich hat (Wolf und Koppel 2017). 
der gering Literalisierten an, häufig oder eher häufig bei der Nutzung des Internets unterstützt zu werden. Zudem trauen sich gering Literalisierte z. B. im Umgang mit Online-Diensten oder bei der Beurteilung hinsichtlich der Glaubwürdigkeit von Texten und Nachrichten weniger zu (Buddeberg und Grotlüschen 2020, 205 f.). Ein nur kleiner Unterschied zwischen gering Literalisierten und der Gesamtbevölkerung besteht allerdings hinsichtlich des Schreibens von Kurznachrichten an eine Person (82,2 \% vs. 89,9 \%) oder an eine Gruppe (41,6 \% vs. 56,3\%).

Auch wenn Lernende im Grundbildungsbereich neben ihren niedrigen schriftsprachlichen Kompetenzen tendenziell niedrigere Fähigkeiten im Umgang mit digitalen Medien aufweisen als die Gesamtbevölkerung, können die meisten nach kurzer Zeit selbstständig mit Computern arbeiten (Goertz und Radomski 2015, 8; Koppel 2017, 246 ff). Das führt zu einem gestärkten Selbstbewusstsein (Goertz und Radomski 2015, 21). Schlussfolgernd sind digitale Technologien sowohl als Instrument als auch als Lernziel in Förderungskonzepten für die Grundbildung einzubinden. Aber auch auf der institutionellen Ebene zeigen Untersuchungen in der Erwachsenenbildung, dass der Digitalisierung ein zentraler Stellenwert in der strategischen Ausrichtung der Einrichtungen zukommt (Schmid, Goertz, und Behrens 2018; Sgier, Haberzeth, und Schüepp 2018).

Trotz der diversen, zum Teil oben aufgeführten Vorteile werden digitale Medien im Grundbildungsbereich aber nicht systematisch (Koppel 2018; Scharnberg u. a. 2017, 5) und in der Erwachsenenbildung insgesamt zurückhaltend eingesetzt (Arnold 2014, 157; Reimer 2010, 9), auch wenn die Einstellung gegenüber dem Einsatz digitaler Medien in der Erwachsenenbildung als durchaus positiv zu beurteilen ist (Koppel 2018; Scharnberg u. a. 2017, 5). Erste Ursachen für den zurückhaltenden Einsatz im Bereich der Alphabetisierung und Grundbildung liefert die MenTa-Studie (Goertz und Radomski 2015). Genannt werden mangelnde Ressourcen insbesondere bezüglich IT-Ausstattung, geringe Bekanntheit von passenden digitalen Lernprogrammen, mangelnde Unterstützung z. B. in Form von Weiterbildung oder zeitlicher Entlastung, um digitale Medien einsetzen zu können (vgl. hierzu auch Scharnberg u. a. 2017, 6) sowie die Gestaltung 
der Programme ${ }^{5}$. Zudem existieren hinsichtlich der konzeptionellen Einbindung digitaler Medien in Grundbildungskursen wenig Erfahrungen (Kamin und Meister 2013; Koppel 2018) obwohl sie als Kriterium für lernförderliche Lernkontexte hinreichend belegt sind (vgl. z. B. Breiter u. a. 2013; Gerick u. a. 2017; Kerres 2008, 117; Scharnberg u. a. 2017).

Anhaltspunkte zu Gelingensbedingungen ${ }^{6}$ lassen sich bedingt in eben aufgeführten Studien und umfassender in (Meta-)Studien finden, die im Schulkontext durchgeführt wurden. Modelle zu Einflussfaktoren digitaler Medien im Lehr-Lernprozess (vgl. z. B. Eickelmann 2014; Herzig 2014; Kamin und Meister 2013) zeigen, dass drei Ebenen für die Betrachtung eines erfolgreichen Einsatzes digitaler Medien erkenntnisleitend sind: die individuelle, die prozessuale und die institutionelle Ebene. Für eine systematische Darstellung werden die aktuellen Forschungserkenntnisse im Folgenden anhand dieser drei Ebenen dargestellt.

Auf der individuellen Ebene geben Studien Aufschluss darüber, dass (einflussnehmende) Überzeugungen, Einstellungen und motivationale Orientierungen (Breiter u. a. 2013; Lin, Chen, und Liu 2017; Lipowsky 2006, 48; Staub und Stern 2002) sowie die Fort- und Weiterbildung des Personals (Gerick und Eickelmann 2017, 16; Herzig 2014, 16) einen positiven Einfluss auf den erfolgreichen Einsatz digitaler Medien haben können.

Auf der prozessualen Ebene hinsichtlich der Lehr-Lernprozesse kann der Einsatz digitaler Medien gelingen, wenn Curricula und Materialen entwickelt und konzeptionell verankert werden (Gerick und Eickelmann 2017, 16), was aber bisher nur bedingt umgesetzt wird (Koppel 2018; Scharnberg u. a. 2017, 5). Damit einher geht auch, dass die Potenziale digitaler Medien eher weniger ausgeschöpft werden (Schaumburg 2003). Konkret weist eine Studie darauf hin, dass kürzere Interventionen erfolgreicher sind als länger angelegte Interventionen (Zwingenberger 2009). Hinsichtlich

5 Die Gestaltung des Mediums hat für die hier fokussierte Zielgruppe aufgrund ihrer Heterogenität, der geringen literalen und computerbezogenen Fähigkeiten und der Lernerfahrungen einen hohen Einfluss auf den gelingenden Einsatz (Koppel 2011; 2017). Eine erste umfangreiche Studie zu Usability-Anforderungen im Grundbildungsbereich liefert Koppel (2017).

6 Abgeleitet aus den Forschungsergebnissen wird ein gelingender Einsatz hauptsächlich anhand des Kriteriums Lernförderlichkeit (z. B. Motivationssteigerung, Unterstützung des selbstgesteuerten/-regulierten Lernens, Lernzuwachs/Kompetenzsteigerung und Steigerung der Kooperation) beurteilt. 
der Gestaltung von Computerprogrammen erweist sich als lernförderlich, wenn mehrere Sinnesmodalitäten angesprochen werden (vgl. z. B. Chandler und Sweller 1991; Herzig 2014; Mayer 2005) und die Möglichkeit zur Selbststeuerung besteht (Hattie, Beywl, und Zierer 2013).

Mehrere Studien liefern Hinweise auf Bedingungsfaktoren für die erfolgreiche Integration digitaler Medien auf der institutionellen Ebene (vgl. z. B. Eickelmann 2010; Gerick und Eickelmann 2017; Gerick u. a. 2017; Getto, Hintze, und Kerres 2018; Krumsvik 2008; Schaumburg u. a. 2007). Für einen gelingenden Einsatz wird insbesondere der Zusammenarbeit zwischen Institutionsleitung und Lehrkräften eine tragende Rolle zugeschrieben (vgl. z. B. Breiter 2017; Gerick u. a. 2017; Herzig 2014). Auf der technischen Seite gehören selbstredend eine zeitgemässe IT-Infrastruktur sowie ein pädagogisch ausgerichtetes IT-Management (Gerick und Eickelmann 2017; Herzig 2014) dazu.

Auch wenn die Studien selbst z.T. kritisch zu hinterfragen und nicht pauschal auf das Lernen mit digitalen Medien (vgl. z. B. Breiter u. a. 2013; Herzig 2014, 21) und den Bereich der Grundbildung übertragen werden können, wird deutlich, dass erst die Verbindung von Technologie und Organisationsentwicklungskonzepten eine qualitativ hochwertige, effektive Nutzung digitaler Medien in Schule und Unterricht erzielen kann (SchulzZander 2001) und somit weniger das «Was» sondern stärker das «Wie» Aufschluss über Gelingensbedingungen geben kann (Higgins, Xiao, und Katsipataki 2012, 3). Auf der institutionellen Ebene ist zu beachten, dass sich Erwachsenenbildungsinstitutionen u.a. durch eine deutlich heterogene Finanz- und Organisationsstruktur auszeichnen (Goertz und Radomski 2015; Kamin und Meister 2013, 3).

$\mathrm{Zu}$ berücksichtigen ist bei diesem Forschungsvorhaben, dass sich Erwachsene im Grundbildungsbereich tendenziell durch geringe Selbstwirksamkeitsüberzeugungen und heterogene Lernbiographien, durch die oftmals ein «Patchwork» (vgl. Jäger u. a. 2010) an Kompetenzen hervorgebracht wird, auszeichnen ${ }^{7}$. Die zielgruppenspezifischen Voraussetzungen von Erwachsenen im Grundbildungsbereich erfordern deshalb entsprechende didaktische und methodische Anpassungen, die sich von

$7 \mathrm{Zu}$ Erläuterungen zielgruppenspezifischer Charakteristika von Personen im Grundbildungsbereich vgl. z. B. Bremer und Pape (2016), Koppel (2017) sowie Nienkämper (2015). 
didaktischen Szenarien und dem Methodeneinsatz in der Schule (mitunter stark) unterscheiden können.

Die fokussierte, übergeordnete Fragestellung für das hier vorgestellte Forschungsprojekt lautet:

- Was sind Gelingensbedingungen für den Einsatz digitaler Medien in der Grundbildung?

Der Begriff «Gelingen» umfasst dabei, was von den Befragten als lernförderlich und umsetzbar bewertet wird. Dabei wird sich der Frage auch aus der «anderen Richtung» angenähert, indem nach Herausforderungen und Hemmnissen gefragt wird. Entsprechend lautet eine untergeordnete Forschungsfrage, die schliesslich Schlussfolgerungen über die Gelingensbedingungen ermöglicht:

- Welche Erfahrungen haben Akteure in der Grundbildung mit dem Einsatz digitaler Medien gemacht und mit welchen Herausforderungen waren sie konfrontiert?

Das Projekt ist explorativ angelegt. Im Rahmen dieses Beitrags werden die Ergebnisse der Geco $^{8}$-Studie dargestellt.

\section{Die Geco-Studie}

Die Geco-Studie hatte zum Ziel, Gelingensbedingungen für den Einsatz computerbasierter Lernprogramme im Alphabetisierungs- und Grundbildungsbereich zu erfassen. Computerbasierte Lernprogramme umfassen jegliche Programme (Software, Apps), die der Förderung von gering Literalisierten dienen (können). Fokussiert wird dabei der Einsatz von PCs und Tablets. Die Geco-Studie wurde von der zentralen Forschungsförderung der Universität Bremen finanziert.

8 gefördert durch die zentrale Forschungsförderung Bremen 


\section{$2.1 \quad$ Vorgehen}

Auf Grundlage des aktuellen Forschungsstandes wurde ein Interviewleitfaden entwickelt, der in 8 ExpertInnen-Interviews per Telefon zum Einsatz kam. Grundlegendes Kriterium für die Auswahl der interviewten Personen war, dass sie in einer Bildungsinstitution im Bereich der Alphabetisierung und Grundbildung tätig sind. Für eine erschöpfende Antwort der Forschungsfrage wurden Personen in unterschiedlichen Rollen bzw. Positionen einbezogen. Die interviewten Personen waren auf der Leitungsebene, der konzeptionellen Ebene oder der Kursebene aktiv. Besonders erkenntnisreich für die Fragestellung wurden jedoch die Erfahrungen von Kursleitenden eingeschätzt, weshalb Kursleitende in der Gruppe der befragten Personen überrepräsentiert sind. Der Kontakt wurde über einen Workshop hergestellt, in dem es um den Einsatz digitaler Medien in der Alphabetisierung ging. Interviewt wurden:

- vier Kursleiterinnen (Interviews «KL 1», «KL 2», «KL 3», «KL 4»)

- zwei Sozialpädagoginnen, von denen eine Person primär beratend und lebensbegleitend aktiv ist (Interview «SozPäd 1»), und die weitere Person Projekte leitet, Anträge für Fördermittel stellt, konzeptionell tätig ist und unterrichtet (Interview «SozPäd 2»);

- eine konzeptionell tätige und koordinierende Akteurin, die primär Kurse konzipiert, plant und koordiniert (Interview «Konz»);

- ein Institutionsleiter, der vorwiegend koordinierenden und organisatorischen Tätigkeiten nachgeht (Interview «InstL»).

Die Interviews dauerten zwischen 20 Minuten und 45 Minuten. Ausgewertet wurden die Interviews mit der qualitativen strukturierenden Inhaltsanalyse nach Kuckartz u. a. (2013), da der Fokus der Fragestellung auf einem konkreten Phänomen - den Gelingensbedingungen - gerichtet ist. Der Interviewleitfaden wurde dementsprechend aus dem aktuellen Stand der Forschung generiert. Durchgeführt wurden die Interviews per Telefon. Die Interviews wurden nach Einholung der Einverständniserklärungen und Erläuterungen zum Datenschutz aufgezeichnet und transkribiert. Für die Auswertung wurde die Analysesoftware MAXQDA verwendet. Nach der initiierenden Textarbeit wurden thematische Hauptkategorien gebildet, anhand derer die Transkripte codiert wurden. Anschliessend 
wurden die Textstellen mit den gleichen Codierungen zusammengefasst, um schliesslich die induktive Bestimmung von Subkategorien am Material sowie die Codierung der Transkripte mit dem ausdifferenzierten Kategoriensystem (Haupt- und Subkategorien) vorzunehmen.

\subsection{Ergebnisdarstellung}

Die Darstellung der Ergebnisse der qualitativen Inhaltsanalyse ist gegliedert nach den Hauptkategorien des in der Inhaltsanalyse gebildeten Kategoriensystems. Folgende Hauptkategorien wurden gebildet:

a. Erfahrungen bezüglich des Einsatzes computerbasierter Lernprogramme

b. Weiterbildung von Akteuren der Alphabetisierung und Grundbildung

c. Einstellungen zum Einsatz computerbasierter Lernprogramme

d. Medienausstattung

e. Konzeptionelle Einbindung computerbasierter Lernprogramme

f. Didaktische Aspekte

g. Gestaltung von computerbasierten Lernprogrammen

Im weiteren Verlauf des Beitrags werden zentrale Ergebnisse anhand der Hauptkategorien vorgestellt. Jeder Abschnitt bezieht sich auf eine Hauptkategorie. Die Subkategorien werden in den jeweiligen Abschnitten verarbeitet und aufgrund der Komplexitätsreduktion nicht explizit genannt.

Bereits zu Beginn dieses Beitrags wurde eine grobe Systematisierung (individuell, institutionell, prozessual) vorgenommen, um den Forschungsstand systematisch abbilden zu können. Auch bei der Ergebnisdarstellung wird diese herangezogen, um eine systematische Darstellung zu unterstützen. Die zuerst vorgenommene, offene induktive Codierung des $\mathrm{Ma}-$ terials hat ergeben, dass mit dieser Systematisierung die Sichtweise unter Berücksichtigung der Forschungsfrage nicht eingeschränkt wird. 


\subsubsection{Individuelle Ebene}

Der individuellen Ebene sind Aussagen zugeordnet, die sich auf die Lehrenden sowie die Lernenden direkt beziehen und sowohl deren Erfahrungen und Einstellungen als auch Fortbildungsbedarfe bündeln.

Die Erfahrungen von Lernenden bezüglich des Einsatzes digitaler Medien werden von allen Befragten als heterogen beschrieben. Für einen gelingenden Einsatz im Kurs wird als zu erfüllende Voraussetzung genannt, dass grundlegende Funktionen bei den Lernenden bekannt sind und sie Zeit haben, um Sicherheit im Umgang mit einem Programm gewinnen zu können (Konz, 49; SozPäd 1, 37). Als Herausforderungen werden beschrieben, dass Lernende vorerst grundlegende Kompetenzen im Umgang mit digitalen Medien aufbauen müssen (InstL, 27) und ihnen die Komplexität der Technik verständlich gemacht werden muss (KL 3, 99). Gleichzeitig stellt die mangelhafte Zugänglichkeit zu digitalen Medien für Lernende ein Hemmnis dar (SozPäd 2, 30; SozPäd 1, 41) und es gibt Lernende, die bisher keine Berührungspunkte mit digitalen Medien hatten (SozPäd 2, 44). Hieran zeigt sich die Herausforderung für die Lehrenden, die Binnendifferenzierung im Kurs teilnehmergerecht zu gestalten. Folglich ist die Vermittlung von grundlegenden Kenntnissen digitaler Medien als ein elementares Bildungsziel in der Alphabetisierungs- und Grundbildungsarbeit zu sehen. In Bezug auf die zielgruppenspezifischen Charakteristika ist noch zu diskutieren, ob bzw. welche Voraussetzungen gegeben sein müssen, um digitale Medien als Lernunterstützung in der Grundbildung in die Kurspraxis integrieren zu können. Allerdings ist auch hierbei der Fokus auf jede(n) einzelne(n) Lernende(n) zu legen und an die Kenntnisse und digitalen Praktiken der Lernenden anzuknüpfen. Möglicherweise kann das Konzept «bring your own device» einen konstruktiven Zugang darstellen, um eventuelle Berührungsängste mit einzelnen Medien gar nicht erst aufkommen zu lassen.

Hinsichtlich der Lehrenden werden die Erfahrungen im Umgang mit digitalen Medien - wie auch bei den Lernenden - als heterogen bezeichnet. Als Voraussetzung für den Einsatz digitaler Medien wird beschrieben, dass ein Bedarf an einem kompetenten Umgang mit digitalen Medien besteht $(\mathrm{KL} 3,35)$ und sie sich mit der Technologie und den nutzbaren Programmen auskennen sollten (SozPäd 1, 49). 
Als herausfordernd erweist sich allerdings, dass aufgrund der tiefgreifenden Mediatisierung eine regelmässige Weiterbildung notwendig ist (InstL,27; Konz, 60), die Weiterbildung des Personals aber nicht immer finanziert wird:

«[...] also da haben wir [...] häufig die Aussage, dass die persönliche Weiterbildung des eingesetzten Personals nicht förderfähig ist, dass das dann quasi dem Träger obliegt, also da klafft noch eine Lücke.» (InstL 29-29)

Lehrende sind für ihre Weiterbildungen entsprechend selbst verantwortlich. Diese Herausforderung wird auch von einer Sozialpädagogin bestätigt (SozPäd 1, 50). Hier ist eine Wechselwirkung anzunehmen: Gerade weil den Lehrenden nur eingeschränkt Weiterbildungsmöglichkeiten eingeräumt werden, ist zu vermuten, dass die Grundvoraussetzungen für den Einsatz digitaler Medien nur bedingt vorhanden sind.

Sofern folglich die Voraussetzung für einen gelingenden Einsatz digitaler Medien im Kurs der kompetente Umgang mit eben solchen ist, gibt es hier noch einen Fort- und Weiterbildungsbedarf bzw. eine wahrgenommene Diskrepanz der Lehrenden zwischen den medialen Kompetenzen, die sie haben und denen, die sie benötigen. Interessant wäre es hier, konkrete Fortbildungsbedarfe zu erheben.

Die Einstellungen - sowohl der Lehrenden als auch der Lernenden werden unterschiedlich eingeschätzt: Die Einstellung der Lehrenden zum Einsatz digitaler Medien scheint tendenziell offen und positiv zu sein (z. B. KL 1, 70; SozPäd 1, 45; InstL, 17), wobei eine sinnvolle Balance zwischen der Nutzung digitaler Medien und analoger Formate beachtet werden sollte (KL 3,115). Die konzeptionell tätige Person bezeichnet den Einsatz aufgrund der gesellschaftlichen Entwicklung zudem als dringend notwendig und unabdingbar, um eine gesellschaftliche Teilhabe zu gewährleisten (Konz, 62). Als unterstützend wird beschrieben, dass Lehrende bezüglich des Einsatzes digitaler Medien über Neugier und Motivation verfügen (Konz, 54; KL 3, 35) und aufgeschlossen gegenüber dem eigenen Lernprozess in der Auseinandersetzung mit den neuen Programmen/Medien sein sollten, um darüber methodisch-didaktisch sinnvolle Herangehensweisen zu identifizieren (KL 3, 115). Bezüglich der Technik äussert die konzeptionell tätige 
Person, dass der Einsatz von Tablets im Vergleich zu Computern als wenig zufriedenstellend gesehen wird, da der Bildschirm zu klein sei (Konz, 49).

Die Lernenden scheinen (laut Aussage von Lehrenden) dem Einsatz digitaler Medien z.T. eher skeptisch, scheu (KL 2, 29; KL 4, 23) oder gar ablehnend gegenüber zu stehen (SozPäd 2, 40), insbesondere die «ältere» Generation (KL 2, 29; SozPäd 1, 41). Auch seien Lernende überfordert («das kapier ich nie») (KL 3, 24) und verängstigt im Umgang mit dem PC:

«[es gibt Lernende], die so eine Angst haben [...] etwas [falsch] zu machen, dass sie wie gelähmt vor dem Ding sitzen und dann ihrem Nachbarn sagen, [...] mach du mal». (Konz, 54).

Diese Aussage kann erstens darauf hindeuten, dass die zielgruppenspezifischen Charakteristika einen abwehrenden Umgang bezüglich «Neuem» umfassen können. Allerdings wird auch von gegenteiligen Reaktionen berichtet, indem einige Lernende gegenüber digitalen Technologien sehr aufgeschlossen sind und viel Spass daran haben (KL 3, 97). Zweitens kann die Aussage darauf hindeuten, dass der Lernprozess aus der didaktischen Perspektive nicht ausreichend begleitet bzw. initiiert wird und hier noch kleinschrittiger und «individueller» begleitet werden muss.

Zusammenfassend stehen die Befragten auf der individuellen Ebene dem Einsatz digitaler Medien offen gegenüber und sehen die Notwendigkeit, digitale Medien in den Kursalltag einzubeziehen. Nichtsdestotrotz sind die Erfahrungen mit digitalen Medien heterogen. Der kompetente Umgang von Lehrpersonen mit digitalen Medien wird als wesentliche Voraussetzung genannt, um digitale Medien erfolgreich in das Kursgeschehen zu integrieren. Für die Weiterbildungen des Lehrpersonals stehen allerdings in den meisten Fällen keine finanziellen und zeitlichen Ressourcen zur Verfügung. Lernende bedürfen zudem einer individuellen, didaktisch fundierten Begleitung beim Einsatz digitaler Medien, um Lernprozesse zu unterstützen und Hemmschwellen abzubauen. Hier scheint es grossen Handlungsbedarf zu geben, woraus sich eine wesentliche Gelingensbedingung auf der individuellen Ebene ableiten lässt: Die Lehrkräfte müssen strukturell, finanziell und vor allem inhaltlich darin unterstützt werden, didaktisch fundierte Einsatzszenarien digitaler Medien im Grundbildungsbereich zu kennen und einsetzen zu können. Im besten Fall ist dies durch Fort- oder Weiterbildungsmöglichkeiten zu erreichen. 


\subsubsection{Institutionelle Ebene}

Auf der institutionellen Ebene werden Ergebnisse zur medialen und räumlichen Ausstattung dargestellt.

Hinsichtlich der medialen und räumlichen Ausstattung zeigt sich ein sehr heterogenes Bild. Die Bewertung der Medienausstattung reicht von ausreichend (digitale Medien können in einem befriedigenden Ausmass/ Umfang genutzt werden) bis hin zu recht geringen Möglichkeiten, digitale Medien zu nutzen: Die Zusammenfassung der Aussagen der interviewten Personen ergibt, dass die meisten Institutionen mit Computern und/ oder Laptops ausgestattet sind. Eine Kursleitung berichtet, dass ihr für die Kursdurchführung an einer VHS keine digitalen Medien zur Verfügung stehen $(K L 3,6)$. Über alle einbezogenen Institutionen hinweg stehen Tablets nur in sehr geringem Umfang zur Verfügung, Smartphones werden bisher nicht eingesetzt. Die Nutzbarkeit der Geräte ist allerdings sehr unterschiedlich: In einigen Institutionen stehen Computerarbeitsplätze jederzeit zur Verfügung, in anderen Institutionen sind wöchentliche Zeitfenster für die Nutzung festgelegt, sodass Kursleitende einmal in der Woche pro Kurs (die z.T. ganztags montags bis freitags stattfinden) die Computerarbeitsplätze nutzen können. Die Internetqualität wird von allen Befragten als ausreichend eingeschätzt. Herausfordernd sei allerdings, dass Organisationen «extreme Sicherheitsmassnahmen» haben und somit keine eigenen Dateien über einen externen Datenträger auf das Gerät der Organisation übertragen werden können (KL 3, 101). Herausfordernd sei zudem, dass die technischen Geräte einem schnellen Wandel unterliegen (SozPäd 1, 41) und regelmässig administriert werden müssen (SozPäd 2, 46).

Als Gelingensbedingung wird von allen genannt, dass die digitalen Medien einwandfrei funktionieren müssen und ausreichend Internetbandbreite vorhanden ist. Zudem müsse die Software administrierbar und in das bestehende System integrierbar sein (InstL, 25). Andersherum sei die Frustrationstoleranz gegenüber langsamen Internetverbindungen bzw. Programmen sehr gering (KL 4, 45). Des Weiteren sei es hilfreich, wenn hinsichtlich der Technik verschiedene Auswahlmöglichkeiten zur Verfügung stünden, sodass Lernende selbst entscheiden können, ob sie beispielsweise mit einem Tablet oder einem PC lernen möchten (KL 3, 36) und es verschiedene Präsentationsmöglichkeiten gibt (Konz, 49). 
Die Institutionsleitung berichtet, dass sie über ein anderes Aufgabenfeld (kaufmännische Umschulungen) bereits PCs zur Verfügung hatten und diese für die Alphabetisierung und Grundbildung nutzen können. Wäre diese Medienausstattung nicht vorhanden gewesen, bezweifelt die Institutionsleitung, dass beantragte Finanzierungen für die Alphabetisierung und Grundbildung bewilligt worden wären (InstL, 21). Auch kann die Investition in Technik ein Finanzierungsrisiko darstellen, wenn sie für ein Projekt angeschafft werden muss, das nur ein Jahr läuft (InstL, 27).

Zusammenfassend wird bezüglich der institutionellen Ebene die Notwendigkeit einer einwandfrei nutzbaren Technik berichtet, die Ausstattung wird als ausreichend beschreiben, jedoch wird das geringe Mass an Flexibilität in der Einbindung der digitalen Medien als Hemmnis und Herausforderung bezeichnet.

\subsubsection{Prozessuale Ebene}

Die prozessbezogene Ebene umfasst Äusserungen zur konzeptionellen Einbindung, zu didaktischen Herangehensweisen sowie zur Gestaltung von Programmen.

Die konzeptionelle Einbindung digitaler Medien wird unterschiedlich realisiert und die Aussagen beziehen sich auf die organisatorische Ebene bis hin zu Konzepten, in denen konkrete Inhalte angegeben sind. Somit werden in einer Institution in Medienkonzepte für Alphabetisierungs- und Grundbildungskurse organisationale und finanzielle Vorgaben einbezogen (InstL, 43-48). Von einem inhaltsbezogenen Konzept berichtet eine Person: «ich habe mich also quasi in der Konzeption festgelegt, dass ich mit diesem „ich-will-lernen.de” arbeite, [...]. Wir haben auch mal so Fördereinheiten drinnen wo wir jetzt die Medienkompetenz stärken, da ist ja meiner Fantasie keine Grenze gesetzt.» (KL 1, 20)

Die Nutzung eines bestimmten Programms hat die Person (KL 1) selbst festgelegt. Fördereinheiten zur Stärkung der Medienkompetenz scheinen allerdings kursübergreifend vorgegeben zu sein, für dessen Umsetzung Interpretationsspielraum zur Verfügung zu stehen scheint und «der Fantasie keine Grenzen» gesetzt sind. 
Die Institutionsleitung berichtet, dass lediglich der organisatorische und infrastrukturelle Rahmen vorgegeben ist, die Inhalte werden von der Kursleitung selbst bestimmt (InstL, 15).

Den Mehrwert des Einsatzes von Lernprogrammen sieht die Leitung insbesondere in einem Ressourcenersparnis, da Akteure der Grundbildung parallel «planerische Sachen» machen können (InstL, 17). Ein ungenutztes Potenzial sieht die Leitung darin, Lehrbücher stärker mit dem Einsatz digitaler Medien zu verknüpfen (InstL, 35). Eine weitere Person berichtet, dass konzeptionell vorgegeben ist, mit welchem Programm gearbeitet werden soll $(K L$ 1, 20) und zu welcher Uhrzeit $(K L 1,72)$ es genutzt wird. Zudem berichten Personen weniger über bestehende institutionell verankerte Konzepte, sondern über grundsätzliche didaktische Prinzipien, die umgesetzt werden, wie beispielsweise Binnendifferenzierung $(K L 1,8)$. Auch wenn die konzeptionelle Verankerung wenig ausformuliert ist, wird betont, dass der Einsatz digitaler Medien für die Lernenden immer in einem sinnvollen, nachvollziehbaren Zusammenhang zu ihrem Lernen und Leben stehen müsse (KL 3, 37). Herausfordernd sei dabei die Verknüpfung von analogen und digitalen Medien:

«Wenn ich das Lehrwerk auf der einen Seite habe, mit dem ich siebzig Prozent meines Unterrichts plane und ein anderes digitales $\mathrm{Me-}$ dium auf der anderen Seite für die anderen dreißig Prozent, habe ich eine Schnittstellenproblematik und Schnittstellen machen immer Aufwand. Und zudem, diesen Aufwand müssen dann sowohl Dozent als auch Bildungseinrichtung bereit sein zu zahlen. Das ist aus meiner Sicht nicht immer der Fall». (InstL, 35)

Gehemmt wird der konzeptionelle Einsatz digitaler Medien dadurch, dass die Anreize für einen konzeptionellen Einsatz digitaler Medien fehlen würden und es für Kursleitende weniger aufwendig ist, die Unterrichtseinheiten mit den papierbasierten Lehrbüchern zu gestalten (InstL, 39).

Die Aussagen deuten darauf hin, dass eine Abstimmung zwischen Leitung und den Lehrenden strukturell kaum verankert ist. Der genannte «konzeptionelle Spielraum» kann ein Hinweis darauf sein, dass keine ausformulierten Konzepte existieren, die Lehrenden dadurch keine Orientierungsmöglichkeiten haben und dies womöglich auch zu Hilflosigkeit führen kann. 
Bezüglich der didaktischen Herangehensweise wird als Herausforderung genannt, die komplexe und abstrakte Materie begreiflich zu machen und Navigation, Aufbau sowie Zuordnung von Begrifflichkeiten nachvollziehbar zu erläutern (KL 3, 41-42). Als gelingende Faktoren werden die Schaffung eines Anlasses für die Nutzung des Programms (KL 3, 13) sowie transparent formulierte Ziele genannt (KL 3, 91). Als Vorteil digitaler Medien wird von der Institutionsleitung betont, dass Lernende ihr Lerntempo selbst bestimmen können; gleichzeitig müsse aber darauf geachtet werden, dass Lernende nicht zu sehr abgelenkt werden (InstL, 17).

Zudem sind für einen gelingenden Einsatz eine deutlich langsame Herangehensweise (KL 4, 25), ein modularer, kleinschrittiger Aufbau (InstL, 25; KL 2, 61), häufiges Wiederholen (im genannten Fall tägliches Wiederholen) um eine Gewöhnung aufzubauen (KL 2, 39; KL 3, 38; SozPäd 1, 47), Raum für den selbstständigen Umgang (KL 3, 14) und Feedbackfunktionen (KL 1, 66; KL 4, 41) notwendig. Gleichzeitig ist aber eine individuelle Unterstützung jederzeit zu sichern (KL 1, 48; KL 2, 39; SozPäd 1, 37) und bei Bedarf Hemmschwellen gegenüber dem Umgang mit dem digitalen Lernmedium abzubauen, was mit einem hohen Ressourcenaufwand verbunden und nicht immer zu gewährleisten ist (KL 4, 25- 27). Als ein Erfolgsfaktor wird genannt, dass der Einsatz spielerisch gestaltet wird und die Teilnehmenden Spass haben (KL 1, 38; KL 4, 32), wobei auch angemerkt wird, dass der Umgang mit dem Computer «nicht so spielend leicht wie von Kindern gelernt wird» (KL 3, 20). Als herausfordernd wird zudem beschrieben, dass es kaum didaktische Materialien für die Einführung von Lernprogrammen gebe (KL 2, 62-63; SozPäd 1, 37), den Wissenstransfer von einer «Seite» auf die nächste «Seite» (SozPäd 1, 41) sowie von dem Medium als Kommunikationsinstrument auf natürliche Weise in das alltägliche Leben (KL 2, 61; KL 3, 44) sicherzustellen. Dies deckt sich mit den Erfahrungen der Lehrenden, denen es noch an didaktischen Einsatzszenarien zu mangeln scheint. Besonders herausfordernd wird das Erlernen der Internetnutzung beschrieben - sowohl inhaltlich als auch bezüglich der Datensicherheit (KL 3). Ausserdem wird darauf aufmerksam gemacht, dass sich eine Differenz des Schreibmodus darin äussere, dass Fehler beim Tippen mit der Tastatur auftreten, die beim Schreiben mit Papier und Stift nicht vorkämen (KL 4, 37). Stressinduzierend sei, wenn zu viele Einstellungsmöglichkeiten vorhanden sind (KL 
2, 45), wobei keine Aussagen dazu getroffen wurden, ab welchem Umfang die Einstellungsmöglichkeiten stressinduzierend sind.

Hinsichtlich der Gestaltung von Computerprogrammen wird die Notwendigkeit einer übersichtlichen Gestaltung (SozPäd 1, 25) und eines wiederkehrenden Aufbaus des Interfaces (KL 4, 43) betont. Auch die Möglichkeit, dass sich Programme auf leichte Sprache umstellen lassen und Texte vorgelesen werden, wird als hilfreich für den Kursalltag beschrieben (SozPäd 1, 25). Hinsichtlich des Aufbaus ist gewinnbringend, wenn zu Beginn der Nutzung eines Programms das Interesse der Nutzerin/des Nutzers geweckt wird (SozPäd 1, 27). Zu Frustration kann es führen, wenn bestimmte motorische Techniken für den Schriftspracherwerb (z. B. Silbenbogenziehen) mit dem Computer mit Hilfe einer Maus umgesetzt werden müssen aber der Umgang mit der Maus noch nicht ausreichend geübt wurde (KL 1, 24).

Zusammenfassend zeigt sich auf der prozessbezogenen Ebene, dass der Einsatz digitaler Medien wenig und selten konzeptionell verankert ist. Kursleitende erhalten folglich kaum Unterstützung seitens der Institution, digitale Medien in die eigenen Kurspraxis zu integrieren. Sollte es dennoch ein Konzept geben und beispielsweise die Nutzung eines speziellen Programms vorgeschrieben sein, so gibt es keine (den Interviewpartnern bekannten) weiterführenden didaktischen Hilfestellungen oder Einsatzszenarien, die die Kursleitung bei der Kursgestaltung unterstützen können. Somit werden medienpädagogische Konzepte - sofern vorhanden - auf der Ebene der praktischen Umsetzung formuliert.

\subsection{Diskussion der Ergebnisse}

Die dargestellten Ergebnisse liefern Hinweise auf Gelingensbedingungen des Einsatzes digitaler Medien.

Auf der individuellen Ebene zeigt sich, dass die Einstellungen gegenüber dem Einsatz computerbasierter Lernprogramme bei den Befragten tendenziell positiv sind. Akteure der Alphabetisierung und Grundbildung sehen einen deutlichen Bedarf und zeigen gleichzeitig auch die Bereitschaft hinsichtlich einer kontinuierlichen Weiterbildung, was dem Forschungsstand entsprechend auch von den Befragten als Erfolgsfaktor identifiziert 
wird. Jedoch stehen finanzielle Mittel nur bedingt zur Verfügung bzw. sind finanzielle Mittel strukturell nicht einbindbar. Es zeigt sich hier ein deutliches Spannungsfeld zwischen Bedarf an Weiterbildung und mangelnden strukturellen finanziellen Ressourcen.

Den Lernenden wird eine heterogene Einstellung attestiert. Dies kann unter anderem an den geringen Erfahrungen liegen, die Lernende mit Computern per se und insbesondere mit computerbasierten Lernprogrammen vorweisen. Es ist anzunehmen, dass die tendenziell geringen Selbstwirksamkeitsüberzeugungen von Personen mit geringer Literalität und die mangelhafte Zugänglichkeit zu Computern diese Tendenz verursacht und/oder verstärkt.

Auf der prozessualen Ebene wird deutlich, dass der Einsatz computerbasierter Lernprogramme dem Forschungsstand entsprechend konzeptionell kaum verankert ist. Lehrende haben dadurch keine oder nur bedingt eine grundlegende Orientierung hinsichtlich eines flexiblen Einsatzes digitaler Medien. Offen bleibt, warum Konzepte zum Einsatz digitaler Medien bisher nur bedingt existieren und umgesetzt werden. Didaktische Überlegungen zeigen, dass eine kleinschrittige, niedrigschwellige Herangehensweise an ein digitales Lernmedium notwendig ist. Dies kann ein Hinweis darauf sein, dass sich die Ergebnisse der Studie von Zwingenberger (2009) auch im Kontext der Alphabetisierung und Grundbildung bestätigen. Auch wenn kaum unterstützende bzw. begleitende didaktische Materialien bekannt sind, werden doch einige dem Forschungsstand entsprechenden Aspekte genannt, die zu einem gelingenden Einsatz beitragen (modularer Aufbau, eine langsame Heranführung und die sinnvolle Einbindung sowie die Anknüpfung an die Lebenswelt). Es ist anzunehmen, dass die Befragten weniger die Technik per se als Einsatznotwendigkeit sehen, sondern der Technikeinsatz hinsichtlich einer sinnvollen Einbindung in den Kursverlauf bewertet wird. Aufgrund der mangelnden Technikausstattung kann allerdings das Potenzial digitaler Medien hinsichtlich eines flexiblen Einsatzes nicht genutzt werden. Für den bei der Zielgruppe hoch relevanten Abbau der Hemmschwellen scheint insbesondere das Ansprechen mehrerer Sinnesmodalitäten (z. B. mittels einer Vorlesefunktion) sowie eine übersichtliche Gestaltung der Programme beizutragen. 
Entgegen dem Forschungsstad scheint die hier fokussierte Zielgruppe allerdings bedingt von einer Selbststeuerung zu profitieren, da mehrere Auswahl- und Einstellungsmöglichkeiten überfordernd wirken können. Auf der didaktischen Ebene zeigen sich somit deutliche Spannungsfelder zwischen Individualisierung und Standardisierung bzw. zwischen Lernbegleitung und Selbstständigkeit. Für die Begegnung dieser Spannungsfelder sind die Möglichkeiten des flexiblen Medieneinsatzes sowie zielgruppenspezifische pädagogische Kompetenzen auf der einen und medienbezogene Kompetenzen auf der anderen Seite notwendig.

Bezogen auf die institutionelle Ebene zeigt sich auch hier, dass die Institutionen aufgrund mangelnder Ausstattung von den Vorteilen des Einsatzes digitaler Medien (zeitliche und räumliche Flexibilität) nur bedingt profitieren können.

Es ist davon auszugehen, dass gerade die vom Forschungsstand identifizierten Vorteile des Einsatzes digitaler Medien in der Alphabetisierung und Grundbildung - und vermutlich insbesondere bei den Lernenden bei Nichterfüllung zu einem gegensätzlichen Trend in Form von Frustration und sinkender Motivation führen. Aus der Perspektive der Lernenden scheint sich das Potenzial digitaler Meiden bezüglich der Steigerung der Selbstwirksamkeit in das Gegenteil verkehren zu können: Vor dem Hintergrund, dass Lernende z.T. Angst haben, etwas an der Technik kaputt zu machen, ist anzunehmen, dass Lernende einen Zusammenhang zwischen ihren eigenen Kompetenzen und einem nicht funktionierenden Lernprogramm sehen können.

Hinsichtlich der Medienausstattung deutet die Aussage der Institutionsleitung auf eine bisher in der Forschung noch nicht thematisierte Abhängigkeit hin: Die Institutionsleitung sieht für die erfolgreiche Drittmitteleinwerbung für Alphabetisierungs- und Grundbildungskurse eine bereits vorhandene Medienausstattung als Voraussetzung. Hier zeigt sich erneut, dass eine ausreichende Medienausstattung eine verbindliche und grundlegende Bedingung für erfolgreiche Alphabetisierungs- und Grundbildungsarbeit darstellt. 


\section{Schlussfolgerungen: Gelingensbedingungen für den Einsatz digitaler Medien im Alphabetisierungs- und Grundbildungsbereich}

Auf der Grundlage der Ergebnisse kann die Forschungsfrage, was Gelingensbedingungen für den Einsatz digitaler Medien in der Alphabetisierung und Grundbildung sind, folgendermassen beantwortet werden: Die empirischen Befunde der hier vorgestellten Studie spiegeln zusammengefasst den Forschungsstand zum Einsatz digitaler Medien in Lehr-Lernkontexten wieder, jedoch sind Gelingensbedingungen identifizierbar, die speziell auf den Alphabetisierungs- und Grundbildungsbereich zutreffen. Diese scheinen zum einen in den spezifischen Charakteristika von Erwachsenenbildungsinstitutionen (heterogene Finanz- und Organisationsstrukturen sowie heterogene Bildungsbiographien von ErwachsenenbildnerInnen) und zum anderen in den zielgruppenspezifischen Charakteristika von gering Literalisierten begründet zu sein.

Auf der individuellen Ebene tragen zu einem gelingenden Einsatz bei:

- ausreichende Erfahrungen im Umgang mit digitalen Medien und Computerprogrammen insbesondere seitens der Kursleitenden,

- die Bereitstellung von finanziellen, zeitlichen und strukturellen Ressourcen für die Weiterbildung der Lehrkräfte,

- pädagogische, zielgruppenspezifische Kompetenzen der Lehrenden, um eine individuelle Unterstützung für Lernende umsetzen zu können,

Hier zeigt sich, dass den genannten Herausforderungen mit systematischer Professionalisierung der Lehrkräfte begegnet werden könnte, um Gelingensbedingungen erfüllen zu können.

Auf der institutionellen Ebene beziehen sich die Gelingensbedingungen vordergründig auf die Technikausstattung. Voraussetzungen für einen gelingenden Einsatz sind

- eine funktionierende Technik und fehlerfreie, administrierbare Software sowie

- die Möglichkeit der flexiblen Nutzung der digitalen Medien. 
Hinsichtlich der prozessbezogenen Ebene tragen vordergründig konzeptionelle und didaktische Hinweise zu einem gelingenden Einsatz digitaler Medien bei:

- die Überführung aktueller Erkenntnisse bezüglich des Einsatzes digitaler Medien in Konzepte (über die Nennung konkreter Softwareprogramme bzw. computerbasierter Lernprogramme hinaus),

- die konzeptionelle Einbindung digitaler Medien,

- die Verzahnung von papierbasierten Lehrbüchern und computerbasierten Lernprogrammen,

- Hinweise und Orientierungsmöglichkeiten für Lehrende, um Lernende an die Internetnutzung heranzuführen,

- die Berücksichtigung didaktischer Prinzipien, insbesondere eine modulare und kleinschrittige Herangehensweise, eine Heranführung an digitale Medien und computerbasierte Lernprogramme unter Berücksichtigung der individuellen Ausgangslagen (z. B. Anpassung des Tempos), Raum für eine selbstständige Nutzung der digitalen Medien und Lernprogramme, die Möglichkeit eines spielerischen Umgangs,

- eine übersichtliche Gestaltung der Lernprogramme,

- eingebundene Feedbackfunktionen,

- Einübung motorischer Fähigkeiten (z. B. im Umgang mit der Maus).

Insgesamt zeigt sich, dass einerseits eine recht klare und homogene ${ }^{9}$ Vorstellung davon besteht, was zu einem gelingenden Einsatz beiträgt. Werden diese Vorstellungen in Anforderungen formuliert, klafft andererseits eine deutliche Lücke zwischen den Anforderungen an einen gelingenden Einsatz und den zur Verfügung stehenden Ressourcen. Dabei wird von den Befragten nicht priorisiert, eine qualitativ hochwertige Technikausstattung zur Verfügung zu haben, sondern es wird lediglich eine funktionierende Technik gefordert. Vor dem Hintergrund des durch die Covid19-Pandemie gestiegenen Bedarf an onlinebasierten Lernformaten zeigt sich ein Risiko des Teilhabeausschlusses, das an Virulenz gewinnt. Um das Risiko nicht weiter ansteigen zu lassen sind 1. Strukturelle Bedingungen

9 Zwischen den Aussagen der befragten Personen konnten keine eindeutigen Diskrepanzen festgestellt werden. Den Tätigkeitsfeldern entsprechend äusserte sich die Institutionsleitung vermehrt zu Ressourcen der Bildungseinrichtung im Vergleich zu den weiteren Befragten. 
zu schaffen, die eine Weiterbildung - auch von Honorarkräften - ermöglichen, 2. Zeitliche und finanzielle Ressourcen für die Weiterbildung zur Verfügung zu stellen und 3. eine funktionierende technische Ausstattung zu realisieren.

\section{Reflexion und Forschungsdesiderata}

Die empirischen Befunde geben Aufschluss über Gelingensbedingungen, die der individuellen, der prozessualen und der institutionellen Ebene zugeordnet werden können. Jedoch regten die Interviewfragen nur bedingt an, auch die Ursache (Vorgehen) mit der Wirkung (erfolgreicher Einsatz bzw. nicht erfolgreicher Einsatz) in Beziehung zu setzen. Wie einzelne Aspekte zueinander in einer Ursache-Wirkung-Beziehung stehen und inwieweit der Zeithorizont zur Identifikation von Gelingensbedingungen einbezogen werden kann bzw. werden sollte, geht aus dieser Studie nicht hervor. Auch ist zu berücksichtigen, dass die Interviewteilnehmenden aus einem Workshop heraus akquiriert wurden, welcher das Thema «Einsatz digitaler Medien in Alphabetisierungs- und Grundbildungskursen» behandelte. Insofern ist davon auszugehen, dass die Teilnehmenden des Workshops bereits ein Interesse an dem Thema hatten und sie entsprechend dem Einsatz digitaler Medien in der Alphabetisierung und Grundbildung tendenziell offen und positiv gegenüberstehen. Dies deutet auf eine Positivauswahl der Befragten hin. Allerdings wurden von den Befragten auch diverse Herausforderungen und kritische Aspekte genannt, sodass lediglich ein geringer Einfluss auf den Erkenntnisgewinn aufgrund der Positivauswahl zu vermuten ist.

Zudem wurde die Perspektive der Lernenden nur indirekt und das Verständnis der interviewten Person von Gelingensbedingungen bedingt berücksichtigt.

Für eine tiefere Auseinandersetzung mit der Forschungsfrage sollten daher zukünftig vier Anforderungsbereiche berücksichtigt werden:

1. Erforschung der Ursachen: Für die Identifikation von Gelingensbedingungen ist eine analytische Herangehensweise einzubeziehen, die Aufschluss über komplexe Wechselwirkungen geben kann: Wird der Einsatz digitaler Medien mitunter als Prozess verstanden, kann die Frage 
nach Ursache und Wirkung über die Betrachtung des Outputs (Zufriedenheit, Motivation, Selbstwirksamkeit) bearbeitbar werden, indem positiv bewertete Einsatzszenarien mit der institutionellen, der personellen und der prozessualen Ebene rückgekoppelt werden.

2. Einbezug von Lernenden: Die Gespräche wurden ausschliesslich mit Institutionsleitenden bzw. Lehrenden geführt. Die Sicht der Lernenden konnte nur indirekt einbezogen werden. Da aber auch der Lernerfolg und die Lernmotivation entscheidende Kriterien für einen erfolgreichen Einsatz darstellen, sind auch Lernende mit ihren Sichtweisen einzubeziehen.

3. Analyse des Verständnisses von Gelingensbedingungen: Für ein tieferes Verständnis von Gelingensbedingungen kann auch die Frage darüber aufschlussreich sein, was die interviewten Personen unter Gelingensbedingungen verstehen. Der Begriff ist zum Zeitpunkt der Vorstudie vom aktuellen Diskurs geprägt, der sich primär daran orientiert, was den Lernerfolg und die Lernmotivation steigert. Möglich ist, dass Akteure des Grundbildungsbereichs andere bzw. weitere Kriterien zur Identifikation von Gelingensbedingungen heranziehen.

4. Eine konkrete Evaluation von tatsächlich eingesetzten digitalen $\mathrm{Me}$ thoden: Hierüber kann a) Aufschluss darüber ermittelt werden, welche didaktischen Ansätze und digitalen Tools hilfreich sind und b) wie sich digitale Tools und analoge Materialien zielführend miteinander verknüpfen lassen. Somit können Lehrende aber auch Lernende an die Ressourcen anknüpfen, mit denen Sie ohnehin bereits arbeiten und es lassen sich womöglich Fort- und Weiterbildungspotenziale/-konzepte ableiten.

\section{Literatur}

Arnold, Rolf. 2014. «Digitale Medien in der Weiterbildung - ein Positionspapier». In Digitale Medien in der Schule, herausgegeben von Bardo Herzig und Silke Grafe, 157-60. Deutsche Telekom.

Aufenanger, Stefan, Renate Schulz-Zander, und Dieter Spanhel, Hrsg. 2001. Jahrbuch Medienpädagogik 1. VS Verlag für Sozialwissenschaften, Wiesbaden.

BMBF und KMK. 2016. «Grundsatzpapier zur Nationalen Dekade für Alphabetisierung und Grundbildung 2016-2026». 
Bonna, Franziska, und Barbara Nienkämper. 2011. «Diagnostik nicht ohne Kursleiter/innen - Begründungen, Konzeption und erste Ergebnisse einer Befragung an Volkshochschulen». In Alphabetisierung und Grundbildung, herausgegeben von Birte Egloff und Anke Grotlüschen, 35-52. Münster: Waxmann.

Breiter, Andreas. 2017. «Medienkonzepte der Schule und die Rolle der Schulträger für die Medienentwicklungsplanung». Journal für Schulentwicklung, Nr. 3: 27-30.

Breiter, Andreas, Stefan Aufenanger, Ines Averbeck, Stefan Welling, und Marc Wedjelek. 2013. Medienintegration in Grundschulen. Bd. 73. Schriftenreihe Medienforschung der lfm. Düsseldorf: LfM - Landesanstalt für Medien Nordrhein-Westfalen.

Buddeberg, Klaus, und Anke Grotlüschen. 2020. «Literalität, digitale Praktiken und Grundkompetenzen». In LEO 2018.Leben mit geringer Literalität, herausgegeben von Anke Grotlüschen und Klaus Buddeberg, 197-225. Bielefeld: wbv Publikationen.

Chandler, Paul, und John Sweller. 1991. «Cognitive Load Theory and the Format of Instruction». Cognition and Instruction 8 (4): 293-332.

Couldry, Nick, und Andreas Hepp. 2016. The mediated construction of reality. Cambridge: Polity Press.

Dinkelacker, Jörg, und Aiga Hippel, Hrsg. 2014. Erwachsenenbildung in Grundbegriffen. Stuttgart: Kohlhammer.

Döbert, Marion, Peter Hubertus, und Sven Nickel. 2000. Ihr Kreuz ist die Schrift: Analphabetismus und Alphabetisierung in Deutschland. Bundesverband Alphabetisierung.

Egloff, Birte, und Anke Grotlüschen, Hrsg. 2011. Alphabetisierung und Grundbildung. Münster: Waxmann.

Eickelmann, Birgit. 2010. Digitale Medien in Unterricht und Schule erfolgreich implementieren. 1. Aufl. Münster: Waxmann.

Eickelmann, Birgit. 2014. «Digitale Medien in der Schule - Herausforderungen, Konzepte und Perspektiven».

Ernst, Annegret, und Karsten Schneider. 2009. "Reichweite der Alphabetisieru ngskurse:Ergebnisse einer repräsentativen Befragung anVolkshochschulen». DIE Zeitschrift für Erwachsenenbildung, Nr. 1: 37-39.

European Comission. 2015. «Education and Training 2020. Improving Policy and Provision for Adult Learning in Europe».

Gapski, Harald, Monika Oberle, und Walter Stafer, Hrsg. 2017. Medienkompetenz. Bonn: Bundeszentrale für politische Bildung.

Gerick, Julia, und Birgit Eickelmann. 2017. «Zusammenschau der Ergebnisse des Abschlussberichts der wissenschaftlichen Begleitung der Evaluation des Projekts «Lernen mit digitalen Medien».

Gerick, Julia, Birgit Eickelmann, Gesa Ramm, und Thore-Olaf Kühn. 2017. «Gelingensbedingungen für den Transfer schulischer Innovationen mit digitalen Medien». Journal für Schulentwicklung 21 (3): 8-14. 
Getto, Barbara, Patrick Hintze, und Michael Kerres, Hrsg. 2018a. Digitalisierung und Hochschulentwicklung. Bd. 74. Medien in der Wissenschaft. Münster: Waxmann. https://nbn-resolving.org/urn:nbn:de:O111-pedocs-169832.

Getto, Barbara, Patrick Hintze, und Michael Kerres. 2018b. «(Wie) Kann Digitalisierung zur Hochschulentwicklung beitragen?» In Digitalisierung und Hochschulentwicklung.Proceedings zur 26. Tagung der Gesellschaft für Medien in der Wissenschaft e.V., herausgegeben von Barbara Getto, Patrick Hintze, und Michael Kerres. Medien in der Wissenschaft 74. Münster: Waxmann.

Goertz, Lutz, und Sabine Radomski. 2015. «Mediennutzungs- und Lerngewohnheiten der Zielgruppe von 〈ich-will-lernen.de〉 (MenTa)».

Grotlüschen, Anke, und Klaus Buddeberg, Hrsg. 2020. LEO 2018. Bielefeld: wbv Publikationen.

Grotlüschen, Anke, Klaus Buddeberg, Gregor Dutz, Lisanne Heilmann, und Christopher Stammer. 2019. «LEO 2018 - Leben mit geringer Literalität».

Grotlüschen, Anke, Rudolf Kretschmann Quante-Brandt, Eva, und Karsten Wolf, Hrsg. 2011. Literalitätsentwicklung von Arbeitskräften. Münster: Waxmann.

Hartung-Griemberg, Anja. 2017. «Medienkompetenzförderung in der Erwachsenenbildung bpb». In Medienkompetenz, herausgegeben von Harald Gapski, Monika Oberle, und Walter Stafer, 166-74. Bonn: Bundeszentrale für politische Bildung.

Hattie, John, Wolfgang Beywl, und Klaus Zierer. 2013. Lernen sichtbar machen. Überarb. deutschsprachige Ausg. Baltmannsweiler: Schneider-Verl. Hohengehren.

Helbig, Christian, und Sandra Hofhues. 2018. «Leitideen in der medienpädagogischen Erwachsenenbildung: Ein analytischer Blick». MedienPädagogik: Zeitschrift für Theorie und Praxis der Medienbildung 30 (Erwachsenenbildung): 1-17. https://doi.org/10.21240/mpaed/30/2018.02.26.X.

Herzig, Bardo. 2014. «Wie wirksam sind digitale Medien im Unterricht». Bertelsmann Stiftung. https://www.bertelsmann-stiftung.de/fileadmin/files/BSt/ Publikationen/GrauePublikationen/Studie_IB_Wirksamkeit_digitale_Medien_im_Unterricht_2014.pdf.

Herzig, Bardo, und Silke Grafe, Hrsg. 2014. Digitale Medien in der Schule. Deutsche Telekom.

Higgins, Steven, ZhiMin Xiao, und Maria Katsipataki. 2012. «The Impact of Digital Technology on Learning: A Summary for the Education Endowment Foundation». https://larrycuban.files.wordpress.com/2013/12/the_impact_of_digital_technologies_on_learning_full_report_2012.pdf.

Jäger, Anjuscha, Moritz Müller, Eva Quante-Brandt, und Eva Anslinger. 2011. «Die Philosophie des Förderns: methodisch-didaktische Prinzipien zur Förderung schriftsprachlicher Kopmetenzen junger Erwachsenener und deren Umsetzung». In Literalitätsentwicklung von Arbeitskräften, herausgegeben von Anke Grotlüschen, Rudolf Kretschmann Quante-Brandt, Eva, und Karsten Wolf. Münster: Waxmann. 
Kamin, Anna-Maria, und Dorothee M. Meister. 2013. "Medienpädagogik in Institutionen der Erwachsenen- und Weiterbildung». Herausgegeben von Dorothee M. Meister, Friederike Gross, und Uwe Sander. Enzyklopädie Erziehungswissenschaft Online. https://doi.org/10.3262/EEO18130315.

Kerres, Michael. 2008. «Mediendidaktik». In Handbuch Medienpädagogik, herausgegeben von Uwe Sander, Friederike Gross, und Kai-Uwe Hugger, 116-22. VS Verlag für Sozialwissenschaften. https://doi.org/10.1007/978-3-531-911588_13.

Kerres, Michael. 2009. «Bunter, besser, billiger? Zum Mehrwert digitaler Medien in der Bildung». it - Information TechnologY 44 (4): 187-92. https://doi. org/10.1524/itit.2002.44.4.187.

Koppel, Ilka. 2011. «Computerbasierte Förderdiagnostik: Usability-Anforderungen von Interfaces für funktionale Analphabet/inn/en». bildungsforschung, Nr. 2: 13-37. https://doi.org/10.25539/bildungsforschun.v2io.130.

Koppel, Ilka. 2017. Entwicklung einer Online-Diagnostik für die Alphabetisierung - Eine Design-Based Research-Studie. Wiesbaden: Springer VS. https://doi. org/10.1007/978-3-658-15769-2.

Koppel, Ilka. 2018. «Digitale Medien in der Grundbildung?! Hemmnisse und Gelingensbedingungen in einer heterogenen Bildungslandschaft».

Krumsvik, Rune Johan. 2008. «Situated Learning and Teachers' Digital Competence». Education and Information Technologies 13 (4): 279-90. https://doi. org/10.1007/s10639-008-9069-5.

Kuckartz, Udo, Stefan Rädiker, Thomas Ebert, und Julia Schehl. 2013. Statistik: Eine verständliche Einführung. Wiesbaden: VS Verlag für Sozialwissenschaften. https://doi.org/10.1007/978-3-531-19890-3.

Lin, Ming-Hun, Huang-Chen Chen, und Kuang-Shen Liu. 2017. "A Study of the Effects of Digital Learning on Learning Motivation and Learning Outcome». Journal of Mathematics Science and Technology Education 13 (7): 3553-64. https://doi.org/10.12973/eurasia.2017.00744a.

Lipowsky, Frank. 2006. «Auf den Lehrer kommt es an». Zeitschrift für Pädagogik, Nr. 51: 47-70. http://nbn-resolving.org/urn:nbn:de:0111-opus-73704.

Mayer, Richard E., Hrsg. 2005. The Cambridge Handbook of Multimedia Learning. New York: Cambridge University Press. https://doi.org/10.1017/ CBO9781139547369.

Münstersches Gespräch zur Pädagogik. 2017. Pädagogischer Mehrwert? Herausgegeben von Christian Fischer. Münstersche Gespräche zur Pädagogik. Münster New York: Waxmann.

Nienkämper, Barbara. 2015. Lernstandsdiagnostik bei funktionalem Analphabetismus. Theorie und Praxis der Erwachsenenbildung. Bielefeld: Bertelsmann.

Niesyto, Horst, und Heinz Moser. 2018. Medienkritik im digitalen Zeitalter. München: kopaed. https://www.horst-niesyto.de/band_medienkritik.pdf. 
Pape, Natalie, und Helmut Bremer. 2016. «Adressat/inn/en-, Teilnehmenden- und Zielgruppenforschung». In Handbuch zur Alphabetisierung und Grundbildung Erwachsener, herausgegeben von Mandy Schönfelder, Cordula Löffler, und Jens Korfkamp, 144-64. Münster: Waxmann.

Petko, Dominik. 2006. «Computer im Unterricht: Videobasierte Fallstudien als Medium praxisnaher Lehrerinnen- und Lehrerbildung». MedienPädagogik: Zeitschrift für Theorie und Praxis der Medienbildung 12: 1-30. https://doi. org/10.21240/mpaed/12/2006.05.11.X.

Pietraß, Manuela. 2011. «Digitale Präsenz - der didaktische Mehrwert der Mediengestaltung». Zeitschrift für Pädagogik 57 (3): 338-49. http://nbn-resolving. org/urn:nbn:de:0111-opus-87297.

Pietraß, Manuela. 2014. «Medien». In Erwachsenenbildung in Grundbegriffen, herausgegeben von Jörg Dinkelacker und Aiga Hippel, 150-57. Stuttgart: Kohlhammer.

Reichart, Elisabeth, Thomas Lux, und Hella Huntemann. 2018. «VolkshochschulStatistik - 56. Folge, Arbeitsjahr 2017». https://doi.org/10.3278/85/0019w.

Reimer, Ricarda. 2010. Lernen mit Medien in der Erwachsenenbildung. Enzyklopädie Erziehungswissenschaft Online. Fachgebiet Erwachsenenbildung. Weinheim: Beltz Juventa. https://doi.org/10.3262/EEO16100039.

Sander, Uwe, Friederike Gross, und Kai-Uwe Hugger, Hrsg. 2008. Handbuch Medienpädagogik. VS Verlag für Sozialwissenschaften. https://doi.org/10.1007/9783-531-91158-8.

Scharnberg, Gianna, Anne-Chatrin Vonarx, Michael Kerres, und Karola Wolff. 2017. «Digitalisierung von Erwachsenenbildung in Nordrhein-Westfalen - Herausforderungen und Chancen wahrnehmen». Magazin Erwachsenenbildung. at 11 (30). http://www.erwachsenenbildung.at/magazin/17-30/mebl7-30.pdf.

Schaumburg, Heike. 2003. «Konstruktivistischer Unterricht mit Laptops?» Dissertationsschrift, Berlin. https://doi.org/10.17169/refubium-5867.

Schaumburg, Heike, Doreen Prasse, Karin Tschakert, und Sigrid Blömeke. 2007. Lernen in Notebook-Klassen. Endbericht zur Evaluation des Projekts «1000mal1000: Notebooks im Schulranzen». https://www.researchgate.net/publication/238769876.

Schmid, Ulrich, Lutz Goertz, und Julia Behrens. 2018. Monitor Digitale Bildung. Gütersloh: Bertelsmann Stiftung. https://doi.org/10.11586/2018007.

Schönfelder, Mandy, Cordula Löffler, und Jens Korfkamp, Hrsg. 2016a. Handbuch zur Alphabetisierung und Grundbildung Erwachsener. Münster: Waxmann.

Schulz-Zander, Renate. 2001. «Neue Medien als Bestandteil von Schulentwicklung». In Jahrbuch Medienpädagogik 1, herausgegeben von Stefan Aufenanger, Renate Schulz-Zander, und Dieter Spanhel, 263-81. VS Verlag für Sozialwissenschaften, Wiesbaden. https://doi.org/10.1007/978-3-322-97494-5_17.

Sgier, Irena, Erik Haberzeth, und Philipp Schüepp. 2018. «Digitalisierung in der Weiterbildung». https://alice.ch/fileadmin/Dokumente/Themen/Forschung/ SVEB_Weiterbildungsstudie2017_2018.pdf. 
Staatsministerium Baden-Württemberg. o. J. «Umfassende Maßnahmen zur beruflichen Weiterbildung». Baden-Württemberg.de. Zugegriffen 12. Januar 2021. https://stm.baden-wuerttemberg.de/de/service/presse/pressemitteilung/pid/umfassende-massnahmen-zur-beruflichen-weiterbildung/.

Staub, Fritz C., und Elsbeth Stern. 2002. «The Nature of Teachers' Pedagogical Content Beliefs Matters for Students' Achievement Gains: Quasi-Experimental Evidence from Elementary Mathematics.» Journal of Educational PsychologY 94 (2): 344-55. https://doi.org/10.1037/0022-0663.94.2.344.

Tröster, Monika, und Josef Schrader. 2016. «Alphabetisierung, Grundbildung, Literalität: Begriffe, Konzepte, Perspektiven». In Handbuch zur Alphabetisierung und Grundbildung Erwachsener, herausgegeben von Mandy Schönfelder, Cordula Löffler, und Jens Korfkamp, 42-58. Münster: Waxmann.

Wolf, Karsten D., und Ilka Koppel. 2017. «Digitale Grundbildung: Ziel oder Methode einer chancengleichen Teilhabe in einer mediatisierten Gesellschaft?» Magazin Erwachsenenbildung.at 11 (30). http://www.erwachsenenbildung.at/ magazin/17-30/meb17-30.pdf.

Wolf, Karsten, und Ilka Koppel. 2014. «Pädagogische Online-Diagnostik für funktionale Analphabeten». Alphabetisierung - eine gesamtgesellschaftliche Aufgabe (Argumente und Materialien der Hanns-Seidel-Stiftung), Nr. 94: 9-20. https:// www.hss.de/download/publications/AMZ_94_Alphabetisierung_03.pdf.

Zwingenberger, Anja. 2009. Wirksamkeit multimedialer Lernmaterialien. Bd. 75. Pädagogische Psychologie und Entwicklungspsychologie. Münster: Waxmann. 